\title{
A Case of $\alpha$-Gal-Unrelated Red Meat-Induced Urticaria Treated by Omalizumab
}

\author{
Makoto Kondo Yoshiaki Matsushima Shohei lida Ai Umaoka \\ Takehisa Nakanishi Koji Habe Keiichi Yamanaka \\ Department of Dermatology, Mie University Graduate School of medicine, Tsu, Japan
}

\section{Keywords}

Red meat-induced urticaria $\cdot \alpha$-gal $\cdot \lg \mathrm{E} \cdot$ Beef $\cdot$ Pork $\cdot$ Omalizumab

\begin{abstract}
A 70-year-old healthy woman was referred to our hospital for chronic urticaria. She did not have a history of allergy, asthma, and rhinitis. She was initially diagnosed with $\alpha$-gal-related urticaria based on an episode of delayed-type urticaria after eating red meat. The results of the intracutaneous allergen test for beef and pork were negative. Fluorenzyme immunoassays specific for IgE against $\alpha$-gal, beef, and pork were also negative. She was diagnosed with an $\alpha$-gal-unrelated red meat allergy following the reproduction of urticaria by a food challenge test. The patient was unresponsive to several drugs, including antihistamines or immunosuppressants. However, omalizumab administration suppressed her symptoms. Key Clinical Message: The diagnosis of red meat allergy may require a repeatability test by consuming red meat even though serum $\alpha$-gal IgE antibody might be negative. The $\alpha$-gal-unrelated red meat urticaria may be responsive to omalizumab.
\end{abstract}

(c) 2021 The Author(s).

Published by S. Karger AG, Basel

\section{Introduction}

Sensitization to $\alpha$-gal has been linked to delayed-type urticaria after eating beef and pork. This phenomenon is related to tick bites inducing IgE antibodies against $\alpha$-gal. In such cases, we first alert the patient not to consume beef and pork and also to avoid tick bites as the urticaria can be intractable and resistant to several therapies. We report a case of $\alpha$-gal-unrelated red meat-induced urticaria with a detailed clinical course. 


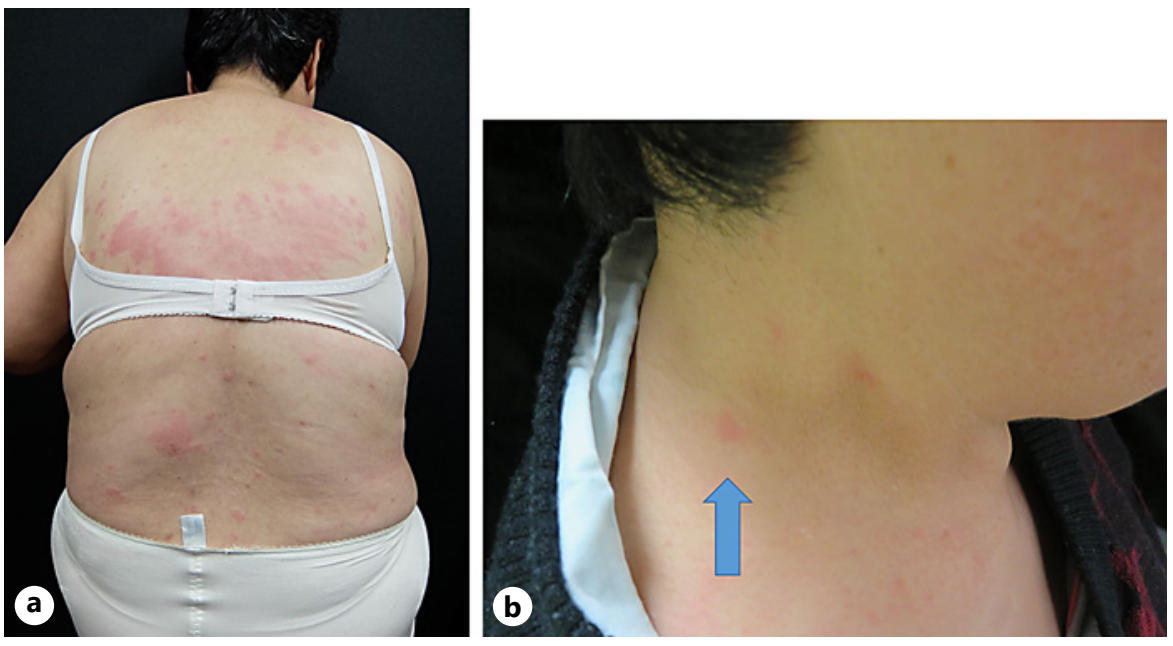

Fig. 1. a Urticaria developed on her whole body at the first consultation. b Arrowhead shows small urticaria welts on the neck.

\section{Case Presentation}

A 70-year-old healthy woman had chronic urticaria for several years. The patient lived near a forest. Her severe symptoms appeared after consuming red meat, including beef and pork. One day after consuming flounder roe, she developed severe urticaria. Upon visiting our emergency room, she received intravenous steroids. She developed urticaria on her entire body without anaphylaxis when she consulted our department (Fig. 1a).

We suspected $\alpha$-gal-related urticaria from episodes of appearance after consuming beef, pork, and flounder roe. Although we advised her not to consume these foods, the urticarial symptoms recurred due to unexpected intake or contact with the restricted foods. One episode of urticaria continued for several days. Her symptoms were unresponsive to various antihistamines and oral steroids; thus, we subcutaneously administered $300 \mathrm{mg}$ of omalizumab. Urticaria disappeared from the day after injection and did not recur until 3 months. We measured serum IgE levels specific to beef, pork, and $\alpha$-gal. Total IgE levels were within the normal range, and $\alpha$-gal-specific IgA, IgG, and IgE levels were less than the measurement sensitivity (Table 1). We repeated the injection of omalizumab (300 $\mathrm{mg}$ ) at a 3 -month interval for the unexpected intake of red meats. Twenty-four days after the second omalizumab administration, we performed a prick test against beef, pork, milk, and normal saline to obtain a definite diagnosis, which yielded negative results. Thirty-eight days after the second omalizumab injection, after obtaining informed consent for the risk of triggering urticaria and anaphylactic reactions, we performed the challenge test for beef. Eight hours after taking beef, she complained of inching and developed urticaria (Fig. 1b). Therefore, we diagnosed her with $\alpha$-gal-unrelated red meat-induced urticaria.

\section{Discussion}

Here, we report a case of urticaria linked to the consumption of beef. Allergic reactions to red meat were suspected when the IgE antibody specific for $\alpha$-Gal is detected [1]. The clinical manifestation of red meat allergy is mostly urticarial, which develops after a few 


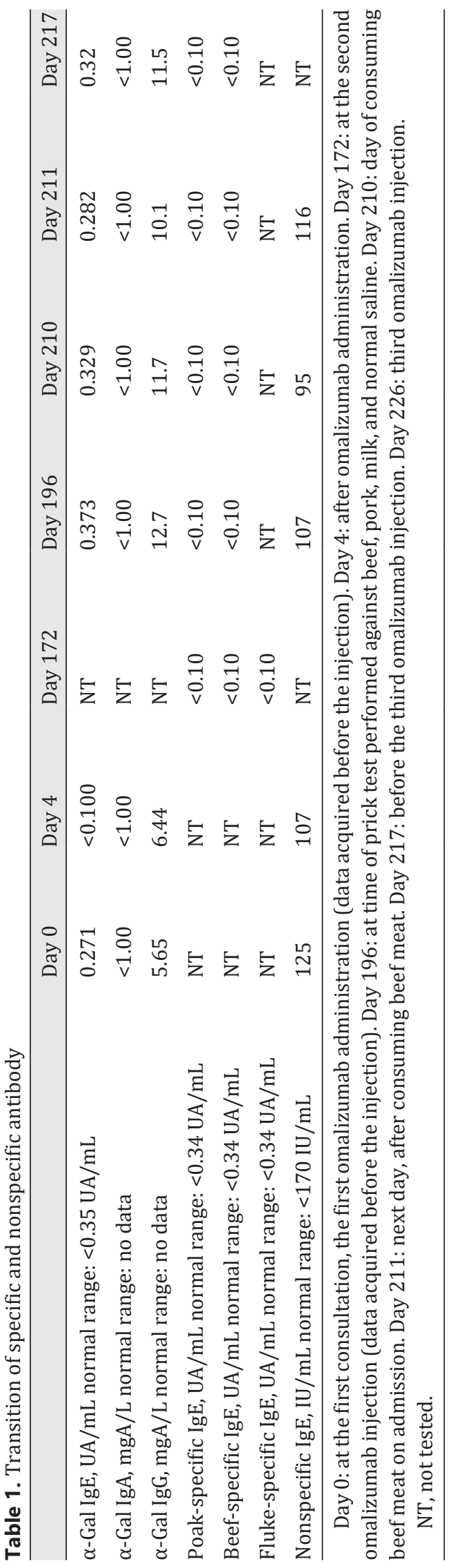

\section{Karger'}


hours of consuming allergy-inducing foods $[1,2]$. We investigated the transition of plasma $\alpha$-gal-specific IgE, IgA, and IgG in the current patient, whose levels were undetectable. Urticaria could be reproduced following a food challenge test. Currently, no relapse has been observed for 2 years with the use of omalizumab. Thus, omalizumab may help treat $\alpha$-galunrelated red meat allergy. A challenge test against red meat may be necessary to confirm the diagnosis when IgE antibodies against $\alpha$-gal are not detected.

\section{Statement of Ethics}

The study was conducted in accordance with the Declaration of Helsinki. The patient provided written informed consent to publish the case, including the publication of images. The study is exempt from ethics committee approval as only 1 case was reported.

\section{Conflict of Interest Statement}

The authors have declared that no competing interests exist.

\section{Funding Sources}

This research received no external funding.

\section{Author Contributions}

Makoto Kondo treated the patient, conceptualization, and investigation. Makoto Kondo, Shohei Iida, Yoshiaki Matsushima, Ai Umaoka, Takehisa Nakanishi, and Koji Habe prepared the manuscript. Keiichi Yamanaka prepared and edited the manuscript.

\section{Data Availability Statement}

The patient data are not publicly available on legal or ethical grounds.

\section{References}

1 Chinuki Y, Ishiwata K, Yamaji K, Takahashi H, Morita E. Haemaphysalis longicornis tick bites are a possible cause of red meat allergy in Japan. Allergy. 2016;71(3):421-5.

2 Kondo M, Nakagawa T, Yamanaka K, Mizutani H. Case with acute urticaria by red meat after Haemaphysalis longicornis bite. J Dermatol. 2017;44(7):e168-9.

\section{Karger' ${ }^{\prime}$}

